\title{
rf amplitude modulation to suppress longitudinal coupled bunch instabilities in the CERN Super Proton Synchrotron
}

\author{
E. Vogel, T. Bohl, and U. Wehrle \\ CERN, Geneva, Switzerland \\ (Received 30 August 2004; published 18 October 2005)
}

\begin{abstract}
Without specific counter measures, the LHC type beam in the SPS suffers from longitudinal coupled bunch instabilities. To prevent them, the SPS impedance has been decreased over the last few years and the operation of a high frequency Landau damping system has been established. In the absence of this Landau damping system one may alternatively introduce an rf amplitude modulation to stabilize the beam. We present results obtained by this method in the SPS and considerations for a potential increase of the longitudinal beam stability in the LHC.
\end{abstract}

DOI: $10.1103 /$ PhysRevSTAB.8.102801

PACS numbers: 29.27.Bd

\section{INTRODUCTION}

In the SPS, LHC type beams are accelerated from 26 to $450 \mathrm{GeV}$. Without specific countermeasures they suffer from both longitudinal single and coupled bunch instabilities. As a result, the bunches show an increased tail population which could lead to losses at injection into the LHC due to uncaptured particles. To overcome these problems, the SPS impedance has been decreased and unused cavities have been removed [1-3]. Microwave instabilities are no longer observed at low energy, but coupled bunch instabilities still appear starting at energies between 200 and $280 \mathrm{GeV}$ and continuing up to high energy. To suppress these latter instabilities a Landau damping rf system is used [2-4]. This system operates with a frequency of $800 \mathrm{MHz}$, which is 4 times the frequency of the main rf system $(200 \mathrm{MHz})$.

The motivation for our study was to try another stabilization method using only the main rf accelerating system. If the beam in the LHC suffers from unexpected longitudinal coupled bunch instabilities [5] such a method could be of great interest, since no higher harmonic system is foreseen.

A Landau damping system increases the incoherent synchrotron frequency spread, the spread of single particles within a bunch. Less particles in a bunch are resonantly driven by external harmonic excitations, for example, beam loading transients caused by other oscillating bunches. The bunches are individually damped.

Alternatively one may increase the coherent frequency spread [6-9]; that is the bunch to bunch frequency spread. Then coupling between the different bunches is reduced and the whole multibunch beam is damped.

According to Sacherer [6], the coherent rms frequency spread $S$ required to damp a coupled bunch phase oscillation, has to be larger than the frequency shift due to the machine impedance. The coupling between the bunches can also be suppressed by an incoherent frequency spread $s$. For phase oscillations the full incoherent spread $(4 \sigma)$ has to be 4 times the frequency shift.
The bunch to bunch frequency spread can be increased by a modulation of the $\mathrm{rf}$ amplitude ( $\mathrm{rf} \mathrm{AM}$ ) locked to the beam revolution. At the SPS this method has already been applied in the past with the proton fixed target beam of $5 \mathrm{~ns}$ bunch spacing and $4 \times 10^{8}$ protons per bunch [10]. LHC type beams have $25 \mathrm{~ns}$ bunch spacing and intensities of $10^{11}$ protons per bunch. rf AM is easily possible as the SPS cavity filling times [11-13] are smaller than the revolution time $(1 \mu \mathrm{s} \ll 23 \mu \mathrm{s})$. Every bunch is exposed to a different rf voltage and has therefore its own synchrotron frequency. In [10] the rf amplitude was modulated sinusoidal whereas we applied a linear rf amplitude change.

\section{CALCULATION OF COHERENT SPREAD DUE TO RF AM}

The relative rms synchrotron frequency spread $S / f_{\text {sm }}$ due to an $\mathrm{rf} \mathrm{AM}$ is given by the rms value of the individual synchrotron frequencies $f_{\mathrm{s} j}$ of the bunches

$$
\frac{S}{f_{\mathrm{sm}}}=\sqrt{\frac{1}{N} \sum_{j=0}^{M-1} b_{j}\left(\frac{f_{\mathrm{s} j}}{f_{\mathrm{sm}}}-1\right)^{2}} .
$$

$f_{\mathrm{sm}}$ is the mean value of the synchrotron frequencies $f_{\mathrm{s} j}, N$ the number of bunches and $M$ the number of all bucket positions. The filling schemes are taken into account by the factor $b_{j}$. It is given by

$$
b_{j}= \begin{cases}1 & \text { position } j \text { occupied } \\ 0 & \text { position } j \text { empty, }\end{cases}
$$

with

$$
N=\sum_{j=0}^{M-1} b_{j} .
$$

For LHC type beams in the SPS, $M$ is 924 . To fill the LHC the SPS will accelerate three or four batches of 72 bunches with gaps of 8 bunch positions in between. Each 
batch is injected in a separate cycle from the PS. For the present study we accelerated only one batch.

To calculate the synchrotron frequency of a bunch at bucket position $j$, we recall that it is proportional to the square root of the rf voltage at this position

$$
f_{\mathrm{s} j} \propto \sqrt{V_{j}}
$$

The amplitude modulation of the rf voltage with the shape factor $A M S_{j}$ (amplitude modulation shape) and amplitude $V_{\text {mod }}$ leads to the voltages

$$
V_{j}=V_{\mathrm{m}}+V_{\mathrm{mod}} A M S_{j}=V_{\mathrm{m}}\left(1+V_{\mathrm{r}} A M S_{j}\right)
$$

where $V_{\mathrm{m}}$ is the mean value of the rf voltage.

For the cases of one or four batches and for a linear voltage change from the first to the last bunch, the voltage increases over respectively $M_{\text {mod }}=72$ and $M_{\text {mod }}=312$ bunch positions as follows:

$$
\begin{aligned}
& A M S_{j}=2 \frac{j}{M_{\mathrm{mod}}-1}-1 \text { for } 0 \leq j<M_{\mathrm{mod}} \\
& A M S_{j}=1-2 \frac{j-\left(M_{\mathrm{mod}}-1\right)}{M-\left(M_{\mathrm{mod}}-1\right)} \text { for } M_{\mathrm{mod}} \leq j<M .
\end{aligned}
$$

Finally, the factor $f_{\mathrm{s} j} / f_{\mathrm{sm}}$ in (1) is given by the ratio

$$
\frac{f_{\mathrm{s} j}}{f_{\mathrm{sm}}}=\frac{\sqrt{1+\bar{V}_{\mathrm{r}} A M S_{j}}}{\frac{1}{N} \sum_{j=0}^{M-1} b_{j} \sqrt{1+V_{\mathrm{r}} \overline{A M S_{j}}}} .
$$

Figure 1 shows the dependence of the relative spread $S / f_{\text {sm }}$ resulting from the voltage ratio $V_{\mathrm{r}}=V_{\text {mod }} / V_{\mathrm{m}}$ for both cases. The relative spread is practically the same in both cases due to the short gaps between the batches.

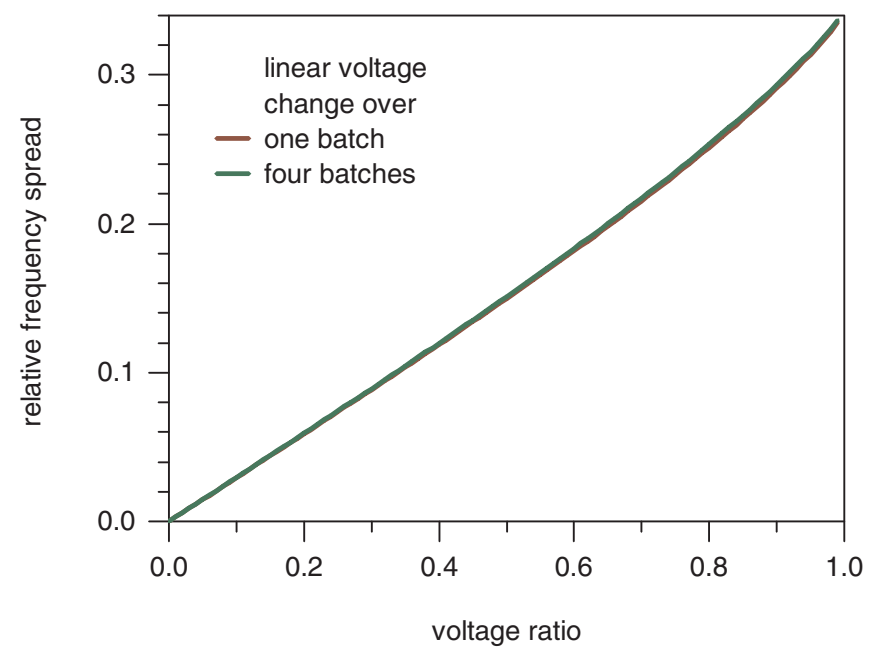

FIG. 1. (Color) Dependence of the relative synchrotron frequency spread in case of one and four batches in the SPS.

\section{INCOHERENT SPREAD SUPPLIED BY THE LANDAU DAMPING RF SYSTEM}

One may estimate the synchrotron frequency shift due to the bunch coupling from the effective accelerator impedance. The coherent frequency spread has to be larger than this value to stabilize the beam.

As the SPS beam is already successfully damped longitudinally by the Landau damping system $(800 \mathrm{MHz})$, we follow another treatment:

The incoherent synchrotron frequency spread is given by the spread between the frequencies at bunch center and the bunch edge $(4 \sigma)$. Assuming a conserved standard emittance of $0.35 \mathrm{eVs}$ (corresponding to the $4 \sigma$ bunch length), these frequencies are first determined for operation with only the $200 \mathrm{MHz}$ rf system and for operation with the $200 \mathrm{MHz}$ and $800 \mathrm{MHz}$ rf systems together [14]. Normalizing the difference values with respect to the small amplitude synchrotron frequencies without $800 \mathrm{MHz}$ results in the additional spread $s / f_{\mathrm{s}}$, supplied by the $800 \mathrm{MHz}$ system. This additional spread is shown in Fig. 2 as a function of the SPS cycle time.

The lower curve in Fig. 2 shows the energy of the SPS for the $21.6 \mathrm{~s}$ long cycle. Beam injection takes place up to $10.8 \mathrm{~s}$. This time is called flat bottom (FB). From 10.8 to $18.2 \mathrm{~s}$ the beam is accelerated to high energy. The period of time at top energy is called flat top (FT).

At FB the SPS shows no coupled bunch instabilities and the $800 \mathrm{MHz}$ system is off. At the beginning of acceleration the $800 \mathrm{MHz}$ voltage is increased and supplies a maximum additional relative incoherent spread of $s / f_{\mathrm{s}}=$ 0.246. It suppresses a bunch coupling having a relative frequency shift of up to one fourth of this value,
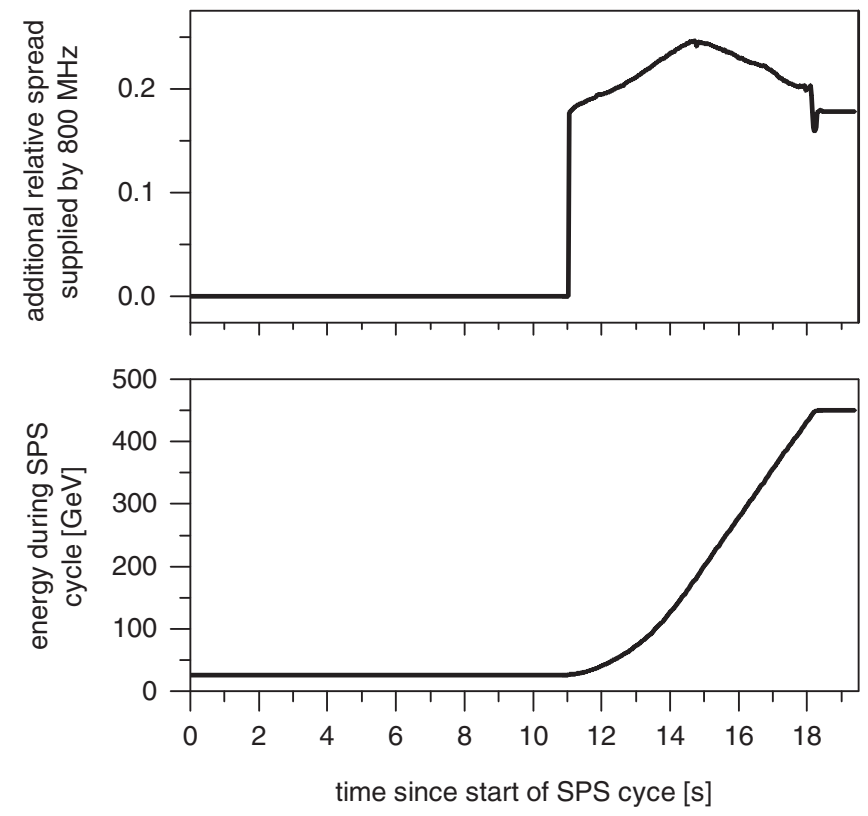

FIG. 2. Additional relative incoherent frequency spread during the SPS cycle, supplied by the $800 \mathrm{MHz}$ rf system. 
$\Delta f_{\text {threshold }} / f_{\mathrm{s}}=\frac{1}{4} s / f_{\mathrm{s}}$ [6]. A relative coherent spread $S / f_{\mathrm{s}}$, larger than this threshold value results in the same stability [6]:

$$
\frac{S}{f_{\mathrm{s}}} \geq \frac{\Delta f_{\text {threshold }}}{f_{\mathrm{s}}}=\frac{1}{4} \frac{s}{f_{\mathrm{s}}}=0.062 .
$$

This value has to be supplied by the rf amplitude modulation. Considering the curves in Fig. 1 it corresponds to a voltage ratio of $V_{\mathrm{r}}=0.22$.

\section{EXPERIMENTAL SETUP}

The experimental setup used for the $\mathrm{rf}$ AM at the SPS is shown in Fig. 3.

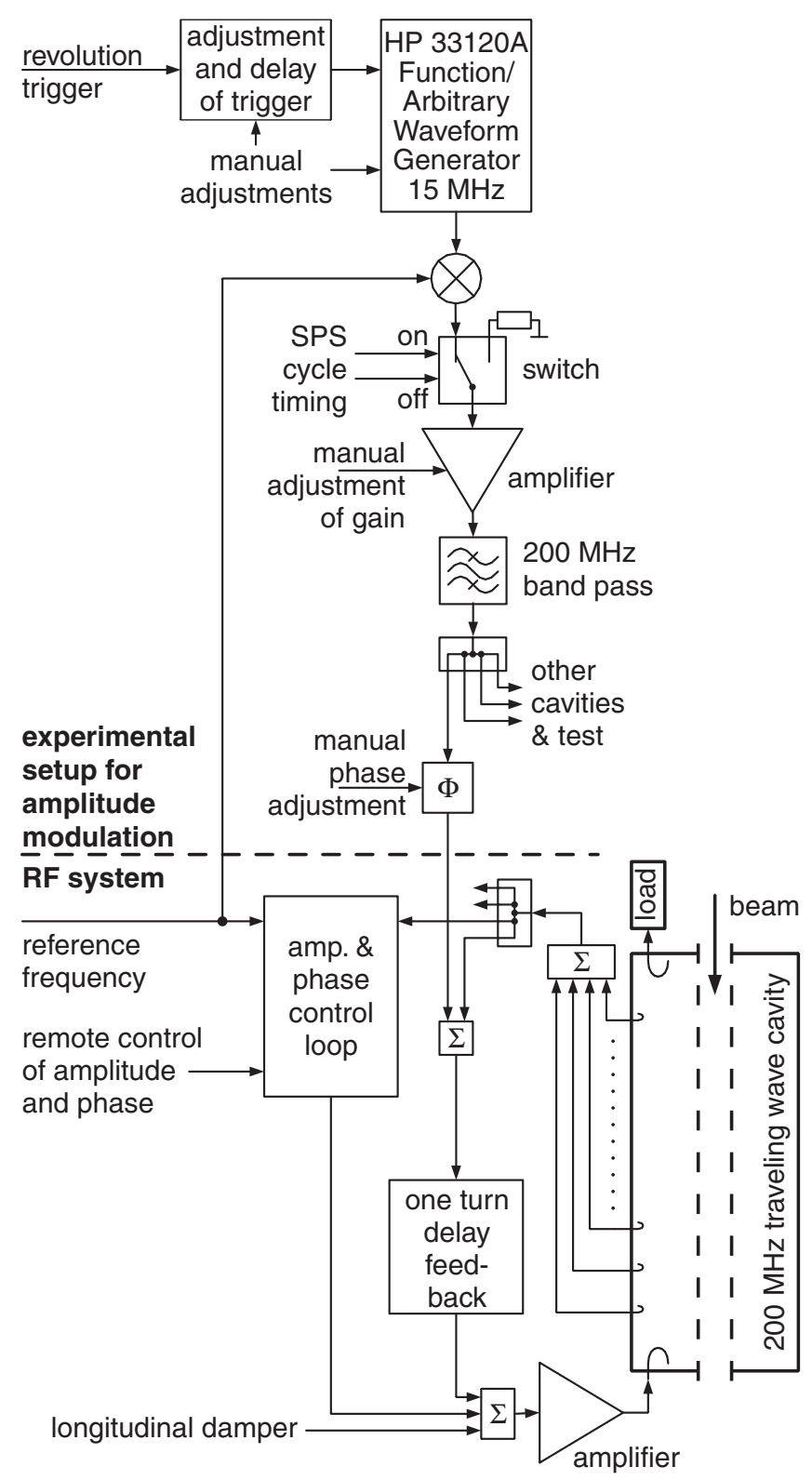

FIG. 3. Experimental setup for the rf amplitude modulation in the SPS.
A waveform generator provides an adjustable trapezoidal output signal triggered at the revolution frequency. Mixing it with $200 \mathrm{MHz}$ results in a $200 \mathrm{MHz}$ rf burst with the trapezoidal envelope. This rf burst is added to the cavity pickup signals used by the one turn delay feedback [15], resulting in a modulation of the cavity voltage. An rf switch in the path of the rf burst disables the modulation at FB and low energies.

For the measurements presented here we set up the rf $\mathrm{AM}$ in the following way:

The $\mathrm{rf}$ frequency changes as the beam velocity increases with increasing particle energy. As a consequence, the phase relation between the rf burst and the cavity $\mathrm{rf}$ changes because of different signal propagation times through the experimental setup and the cavity rf control. At energies above about $64 \mathrm{GeV}$, this effect is negligible and so the timing for the rf switch is set to enable the rf AM only after this energy. This is not a restriction for the suppression of the instabilities as they only appear at energies higher than $200 \mathrm{GeV}$.

The phasing of the rf burst and the cavity rf voltage is adjusted by varying the manual phase shifter, so that the observed modulation amplitude reached its maximum. To protect the final stage rf amplifiers against overcurrent peaks, we chose a phase leading to a reduction in cavity voltage.

By switching off the one turn delay feedback for short times, we could observe the beam loading transients and hence the position of the beam with respect to the modulation waveform. We adjusted the position of the modulation waveform by delaying the revolution trigger for the waveform generator. Afterwards the rise time of the trapezoidal pulse was adjusted so that the whole batch was covered simultaneously by the falling rf edge in all four $200 \mathrm{MHz}$ cavities. These cavities are installed at different places in the SPS and as a result the signal propagation times are slightly different. Consequently the rise time of the pulse had to be longer than the batch.

Figure 4 shows the trapezoidal modulation of the $\mathrm{rf}$ obtained and the position of the accelerated batch.

The rf amplitude and phase control loops adjust the average rf amplitude and phase to their set points within the SPS cycle. To give the maximum possible incoherent frequency spread, the rf voltages are programmed in normal operation so that the bunches fill almost the whole bucket. When operating the rf with AM with the bunches located along the falling rf edge of the modulation, the programmed rf voltage would be reduced giving too small buckets and beam losses. To avoid this we increased the programmed rf voltages so that the average voltage, seen by the bunches, was about nominal. As a result we observed only small losses as in normal operation. Figure 5 shows an example of the programmed rf voltage during the SPS cycle for normal operation and for rf AM operation with the amplitude shown in Fig. 4. 

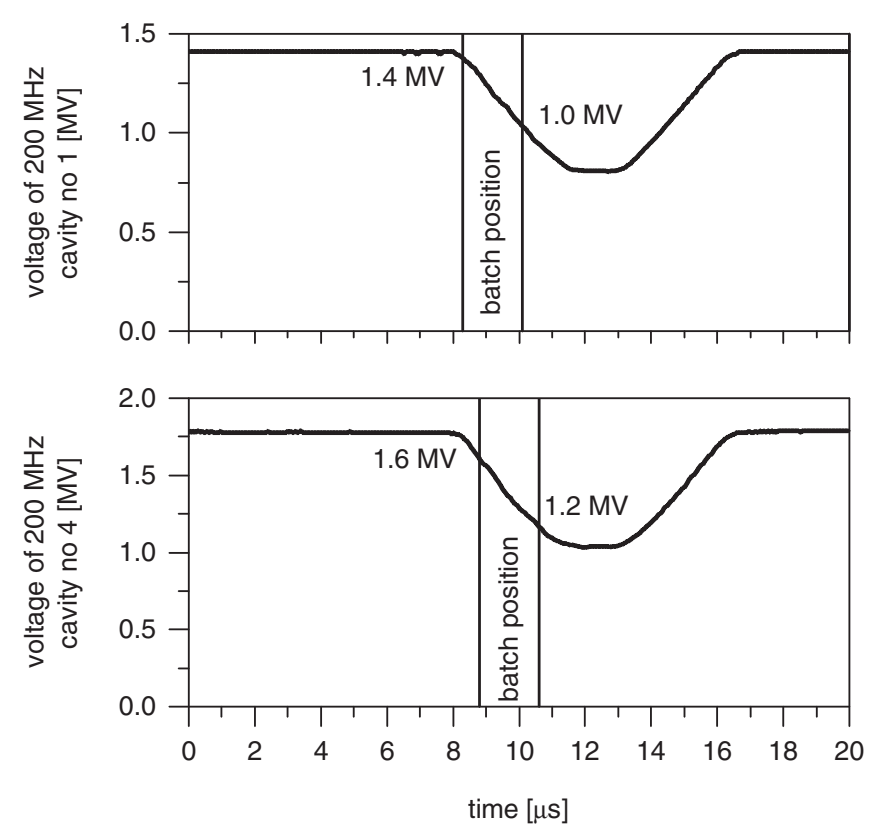

FIG. 4. Measured rf amplitude modulation in $200 \mathrm{MHz}$ rf cavities of the SPS. All four cavities were modulated. SPS revolution time: $23 \mu \mathrm{s}$.

The voltage increase after reaching FT, shown in Fig. 5, is necessary to match the bunches for transfer to the LHC.

Two longitudinal beam control loops are in operation for damping LHC type beams in the SPS:

The phase loop changes the rf frequency as a function of the longitudinal bunch position with respect to the $\mathrm{rf}$

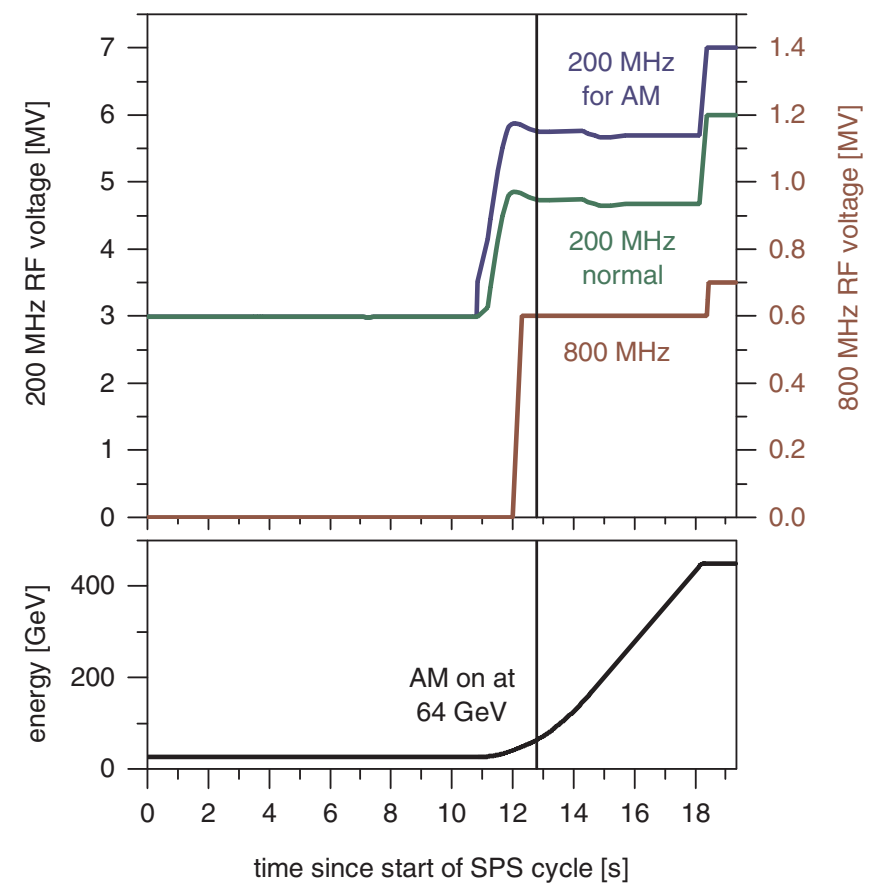

FIG. 5. (Color) rf voltage and energy during the SPS cycle (21.6 s). bucket center [16] and damps the lowest coupled bunch mode $(l=0)$, all bunches oscillating in phase.

In addition there is a longitudinal damper, whose layout is shown in Fig. 6. It feeds back a phase signal shifted by $90^{\circ}$ at the synchrotron frequency. This feedback is able to damp beam phase oscillations having a small oscillation phase shift from bunch to bunch (coupled bunch modes $l \lesssim$ 50). It is used to damp low energy instabilities driven by the rf cavity impedances.

During our studies both loops were always active.

\section{STABILIZATION DUE TO AM}

To observe coupled bunch instabilities in the SPS we used the phase signal of the longitudinal damper, Fig. 6 . The pickup bandwidth is just high enough to separate single bunches of LHC type beams with their bunch spacing of $25 \mathrm{~ns}$. One clearly observes whether the beam is stable or not. In the stable case the signals are smooth along the bunch trains; in case of coupled bunch instabilities one observes ripples.

The first measurements compare three cases: Operation without the $800 \mathrm{MHz}$ Landau damping system and without rf AM, operation with the $800 \mathrm{MHz}$ system and without rf AM and operation without the $800 \mathrm{MHz}$ system but with $\mathrm{rf}$ AM. We accelerated one batch of 72 bunches and the bunch intensity was $0.27 \times 10^{11}$ protons per bunch, that is about one fourth of the nominal LHC intensity of $1.15 \times$ $10^{11}$ protons per bunch.

Figure 7 shows a typical mountain range plot of the measured bunch phase in the case of operation with the $200 \mathrm{MHz}$ system alone. After $16 \mathrm{~s}$ into the cycle, that is at about $280 \mathrm{GeV}$, the beam becomes unstable, observable as ripples on the phase pickup signal.

Figure 8 shows the case when the $800 \mathrm{MHz}$ system is in operation. The ripple observed previously vanished, the beam is stable.

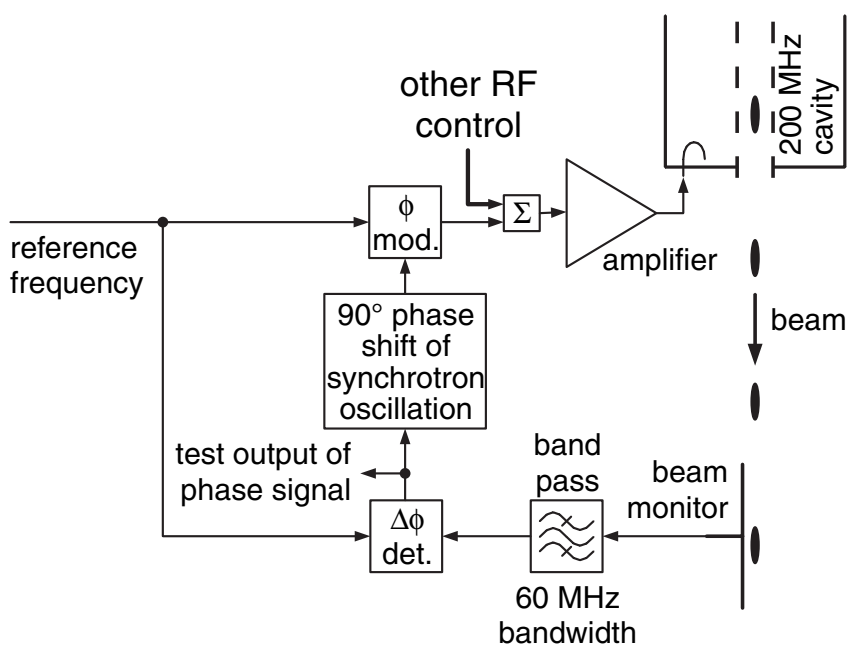

FIG. 6. Scheme of the longitudinal damper. 


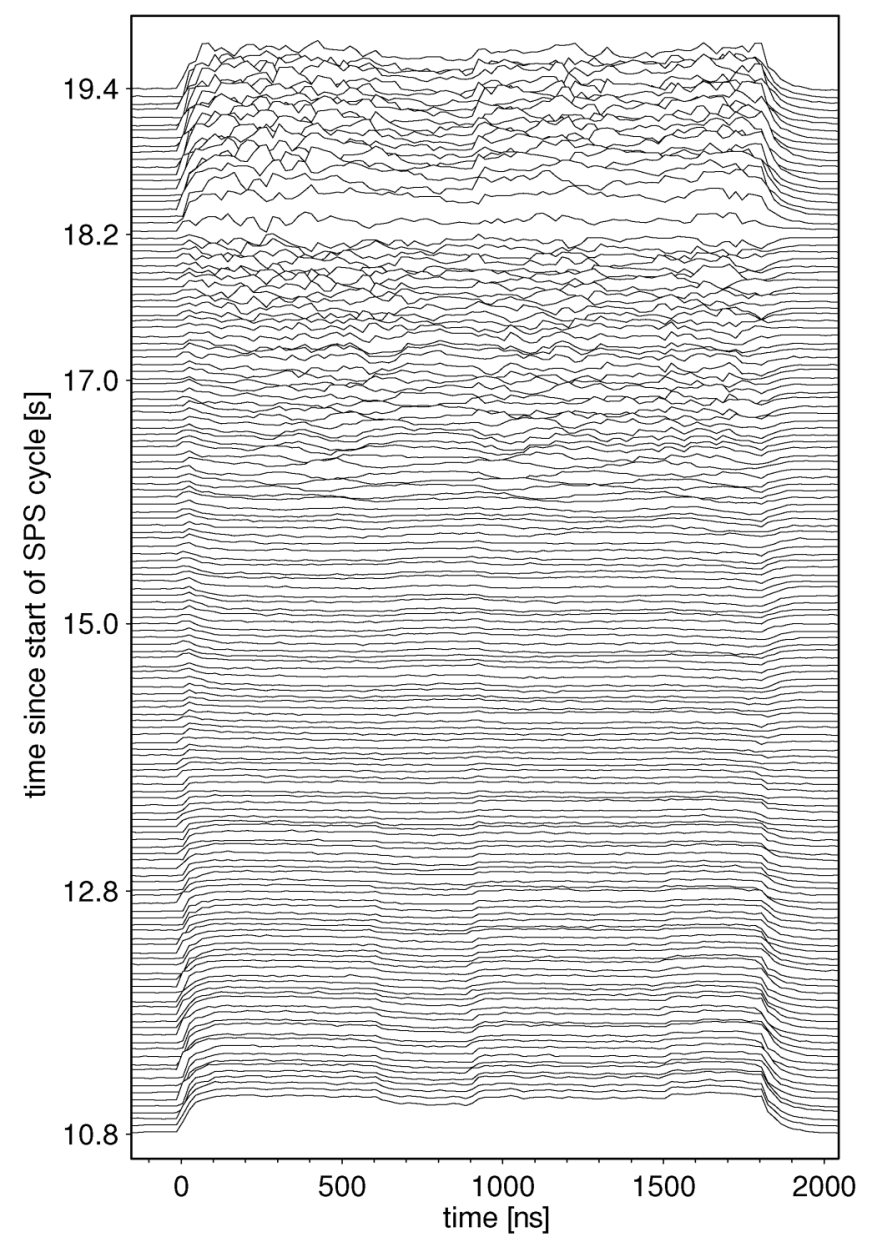

FIG. 7. Mountain range measurement of the pickup signal of the longitudinal damper. The $800 \mathrm{MHz}$ system and the rf AM are switched off.

Finally, Fig. 9 shows the result when the SPS is operated without $800 \mathrm{MHz}$ but with rf AM with a sufficiently high amplitude. The coupled bunch instabilities are also well suppressed.

With $\mathrm{rf}$ AM the beam was stable for a voltage ratio of $V_{\mathrm{r}}=0.16 \pm 0.02$. At the next smaller value measured $V_{\mathrm{r}}=0.12 \pm 0.02$ the beam was unstable.

Afterwards we increased the beam intensity in steps up to $1.01 \times 10^{11}$ protons per bunch. The beam was still stabilized up to FT, but there we observed unstable behavior, Fig. 10. The fact that the beam becomes only unstable at $450 \mathrm{GeV}$ indicates that we are near the intensity threshold for rf AM with the voltage ratio of 0.16 .

Even at high intensities we observed no increased beam losses as compared to normal operation.

\section{LONGITUDINAL BEAM PROPERTIES}

It was also the aim of the study to examine the longitudinal bunch properties under the different conditions. For this purpose we recorded the signal of a resistive gap

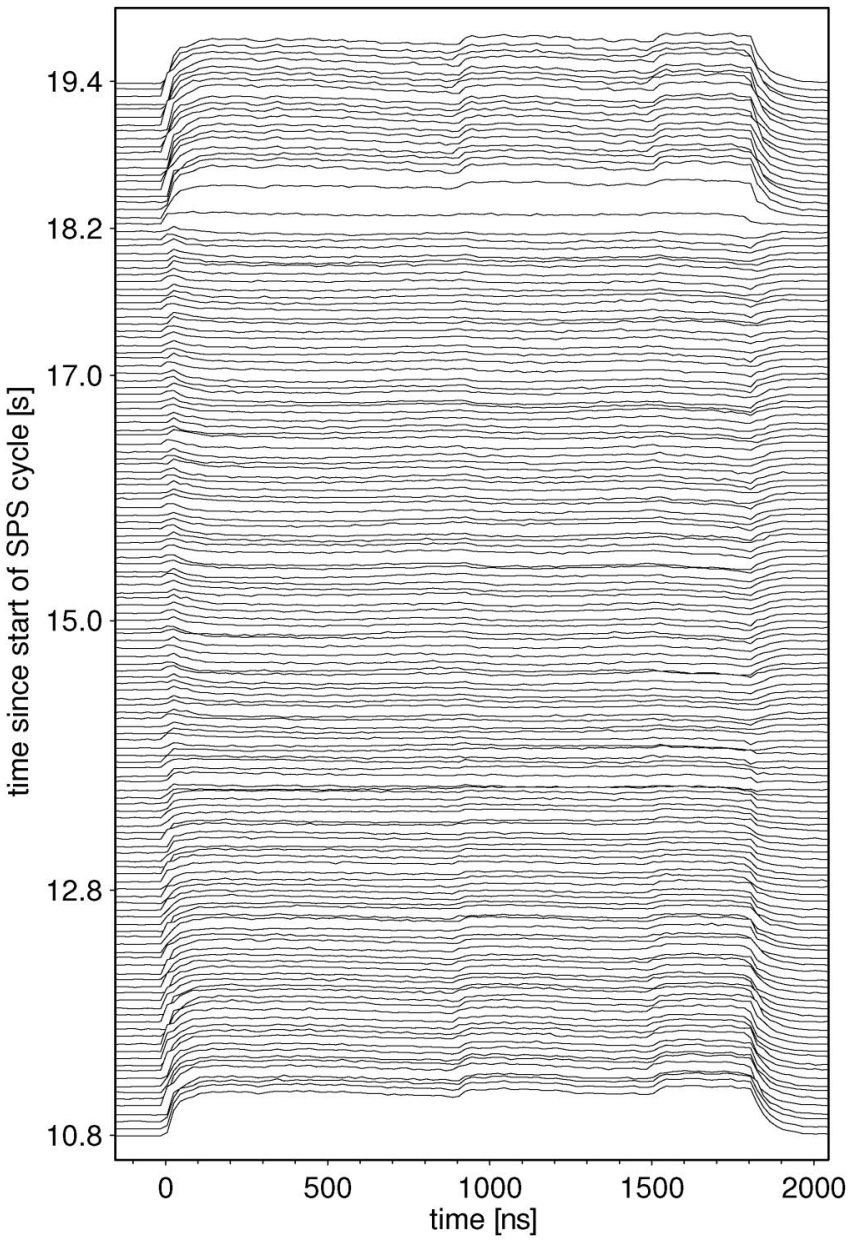

FIG. 8. Mountain range of phase pickup. The $800 \mathrm{MHz}$ system is operating and the $\mathrm{rf} \mathrm{AM}$ is switched off.

monitor via a long coaxial cable of about $200 \mathrm{~m}$. With an oscilloscope of $1 \mathrm{GHz}$ bandwidth and $4 \mathrm{GS} / \mathrm{s}$ sampling rate we recorded the profile of 5 or 50 bunches, respectively. The cable dispersion and the low pass characteristics of the oscilloscope tend to lengthen the bunches. Using measured transfer functions the data was corrected to compensate for this effect. In the following, the $4 \sigma$ bunch length of each bunch is determined by the $\sigma$ value of a Gaussian fit to the acquired data. The measurements presented in this section were taken at bunch intensities of $0.27 \times 10^{11}$ protons per bunch.

We will first have a look at the bunch length within the batch at FB and FT. Figure 11 shows it for the three cases, no $800 \mathrm{MHz}$ and no rf AM, only $800 \mathrm{MHz}$ and only rf AM.

The systematic bunch length variation at low energy, namely, two short bunches, two long bunches, two short bunches and so on, is created in the PS preaccelerator. In all three cases, the bunches show the same length at low energy, as expected.

At high energy the bunches are longest with no additional stabilizing mechanism due to the emittance dilution 


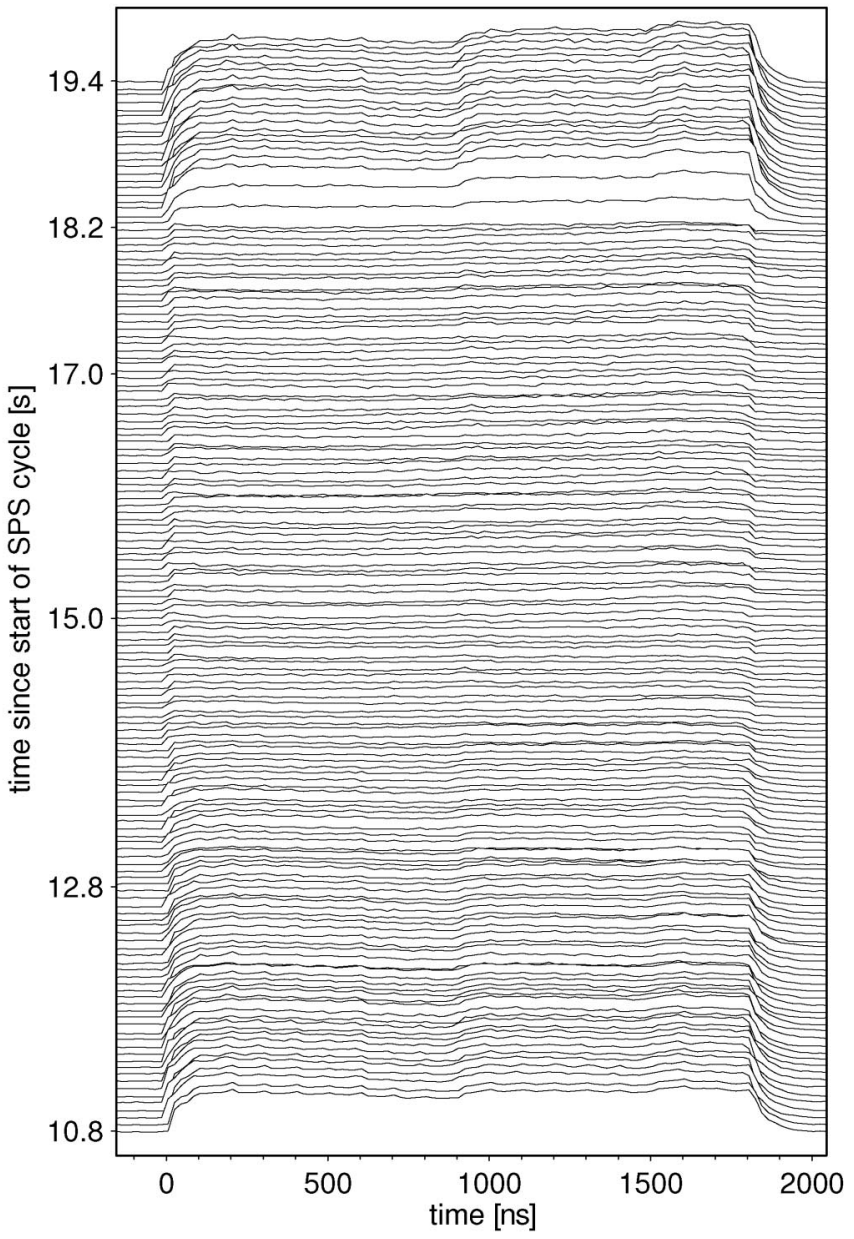

FIG. 9. Mountain range of phase pickup. The $800 \mathrm{MHz}$ system is off and the rf AM is switched on at $12.8 \mathrm{~s}(64 \mathrm{GeV})$.

produced by the instability. The bunches with $800 \mathrm{MHz}$ are shortest: There is no emittance dilution due to an instability and there is also a small additional compression by the $800 \mathrm{MHz}$ system, which is operated in phase with the $200 \mathrm{MHz}$ system (bunch shortening mode [4]). Bunches accelerated with rf AM show a length in between. There is no emittance dilution from an instability but there is no additional compression by a higher harmonic rf system.

For constant longitudinal emittance, the bunch length $l$ is about inversely proportional to the fourth root of the $\mathrm{rf}$ voltage. In the case of of AM one would expect a bunch length change from the first to the last bunch of

$$
\frac{l_{72}}{l_{1}}=\sqrt[4]{\frac{V_{1}}{V_{72}}}=\sqrt[4]{\frac{1+V_{\mathrm{r}}}{1-V_{\mathrm{r}}}},
$$

where $V_{1}$ and $V_{72}$ are the voltages, seen by the first and the last bunch. As Fig. 11 shows only 50 bunches out of 72, we have to take additionally into account the factor 50/72. Then we would expect to observe a bunch length variation of $6 \%$ (for $V_{\mathrm{r}}=0.16$ ). However, the bunches seem to show

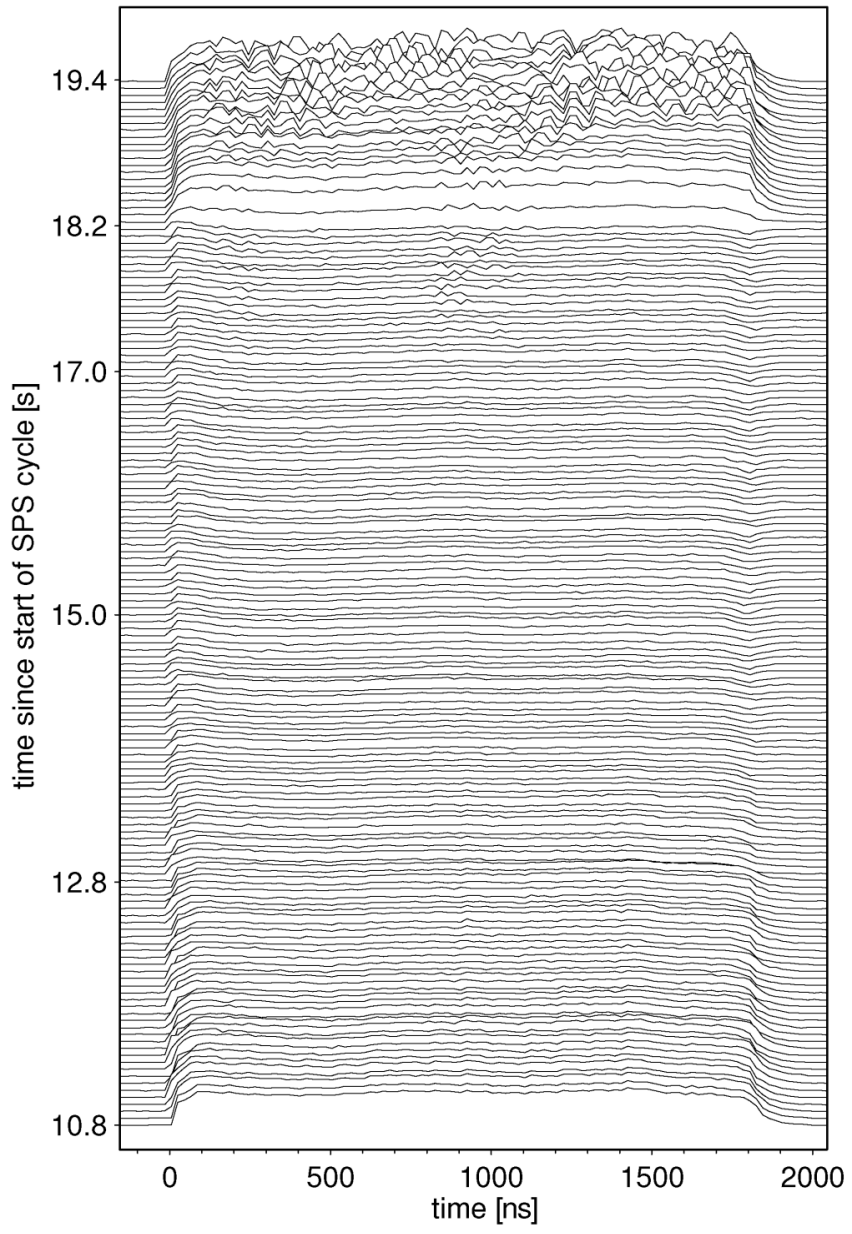

FIG. 10. Mountain range of phase pickup at high beam intensities. Operation with rf AM and without $800 \mathrm{MHz}$.
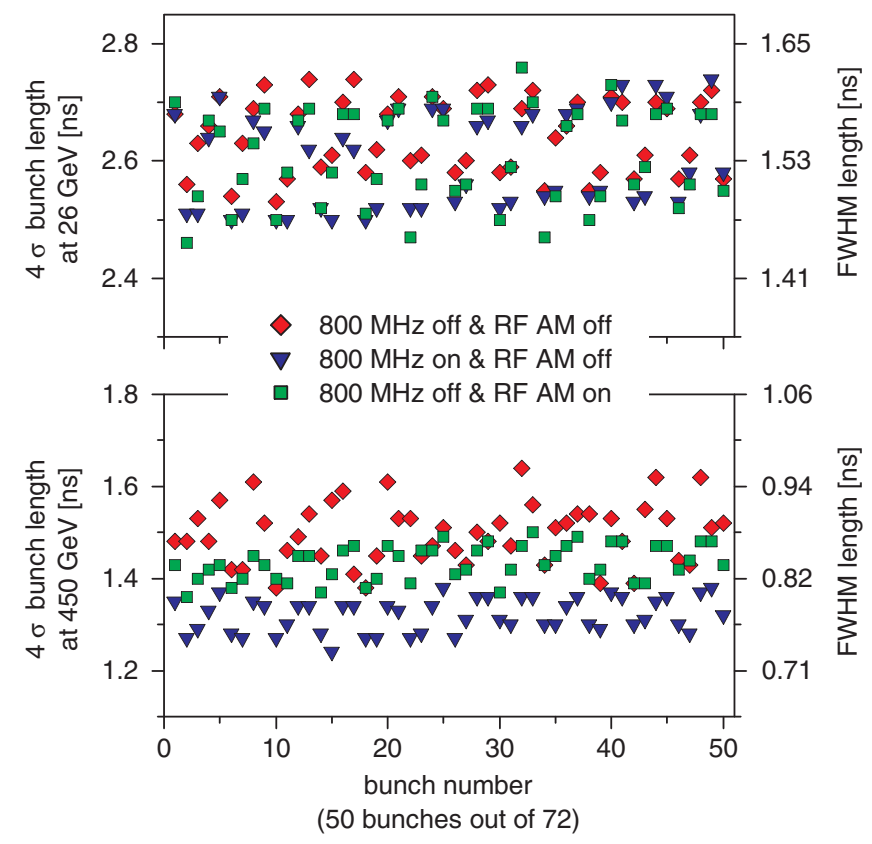

FIG. 11. (Color) Bunch lengths measured at FB and at FT. 
no significant additional length variation over the batch from the rf AM.

To examine this effect in more detail we applied a linear fit to each acquisition of 50 bunch lengths versus bunch position. The ratios of the fit values for the last and first bunch position are shown in Fig. 12.

In the case of operation with the $200 \mathrm{MHz}$ system only and also in the case of the $200 \mathrm{MHz}$ together with the $800 \mathrm{MHz}$ system, we observe no significant bunch length variation. The short negative excursion of the values near $17 \mathrm{~s}$ in case of an operation with $200 \mathrm{MHz}$ only is believed to be an artifact, caused by an oscillating beam. Operating the SPS with rf AM the bunch length ratio is about $6 \%$ after switching the AM on. This is the expected value. It decreases slightly during the continuing acceleration and disappears rapidly at the end of the acceleration process. Hence, there are effects which increase the bunch length to similar values. This means that the emittance dilution depends on the bunch length or the incoherent frequency spread. Reasons for the fast reduction of the bunch length ratio at the end of the acceleration process may be the sudden change of the synchronous phase from $26^{\circ}$ to zero and an increase of the rf voltage at the end of acceleration.

The tendency of a beam when blowing up to acquire similar bunch lengths, despite different voltages due to an rf AM, was also observed at the HERA proton ring [17].

Figure 13 shows the bunch length development of the last bunch during acceleration. At low energy the bunch shows in all cases a length of about $2.5 \mathrm{~ns}$. Without

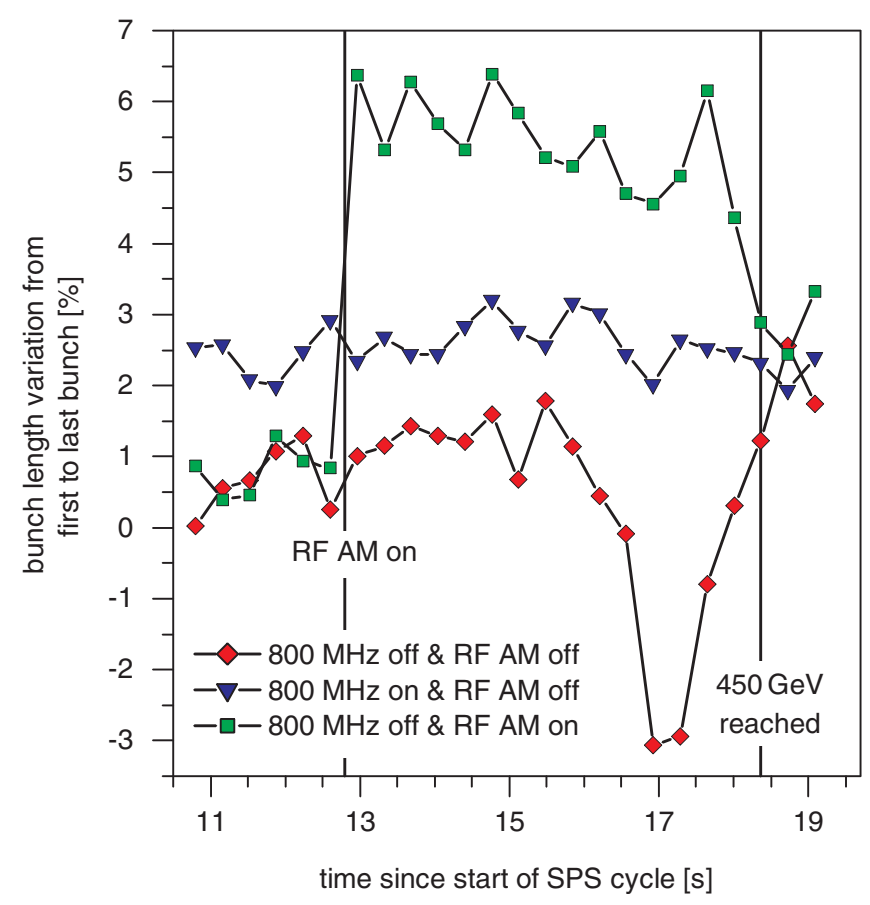

FIG. 12. (Color) Variation of bunch length ratio of first and last measured bunch within the SPS cycle.

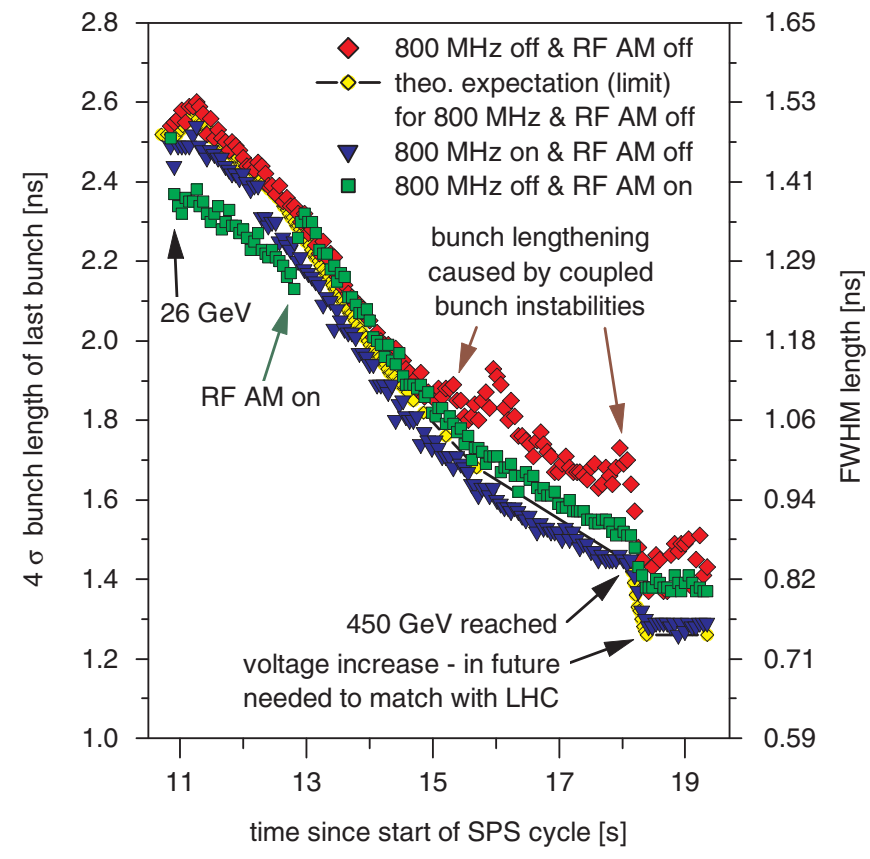

FIG. 13. (Color) Bunch length development of last bunch.

$800 \mathrm{MHz}$ and without rf AM the bunch becomes unstable near $15.5 \mathrm{~s}$ in the SPS cycle ( $\approx 250 \mathrm{GeV})$, indicated in the plot by the scatter of bunch length values. Accelerating with $800 \mathrm{MHz}$ or with $\mathrm{rf} \mathrm{AM}$ the bunch length evolution through the cycle becomes smooth. The bunch with $800 \mathrm{MHz}$ is shorter than the bunch with rf AM.

The bunch with of AM is compressed at the start of acceleration due to the above mentioned $\mathrm{rf}$ trim for $\mathrm{rf}$ AM operation before switching the AM on. The voltage was too high during this time. After switching the AM on, the bunch length returns to the values obtained otherwise. We note that this unnecessary compression at lower energies was reversible and had no further influence on the beam stability.

Figure 13 also shows the expected bunch length for a bunch with an emittance of $0.36 \mathrm{eVs}$ (corresponding to the $4 \sigma$ bunch length) accelerated without $800 \mathrm{MHz}$ and without rf AM. One observes in each case an increase in the difference between the measured bunch length and the expected one. Hence, we have a slight emittance blow up. About one half of the total blow up takes place towards the end of the acceleration and at the final increase of the rf voltage. We recall that at the same time we observe the sudden disappearance of the bunch length slope in the case of the rf AM.

\section{APPLICATION TO THE LHC}

At the LHC direct modulation of the rf amplitude is feasible [18]. The maximum available voltage ratio is restricted by the long filling times of the superconducting cavities. 
In the case of broad band impedances, bunches with nearly equal synchrotron frequencies will couple, even if distant bunches have different synchrotron frequencies. Using sinusoidal modulation leads to relatively large zones at the turning points of the modulation where successive bunches have comparable synchrotron frequencies and may couple. These zones are significantly reduced with triangular modulation.

Ideal, sharp cusps are not possible due to rf power and bandwidth restrictions. Triangular shapes with rounded cusps should be possible in the LHC for voltage ratios of $V_{\mathrm{r}}<0.143[18,19]$. The maximum ratio can be obtained only if no rf power is needed for transient beam loading compensation. Therefore, with beam the voltage ratio has to be smaller.

Shorter bunches cause stronger wake fields than longer ones and, as a result, they couple more strongly to each other. If one operates near the instability threshold bunches at the maximum voltage couple first and drive after some time the remaining bunches [17]. This effect is minimized by placing the area of maximum voltage in the beam dump gap.

Figure 14 shows a possible voltage shape for LHC taking into account the bunch pattern. Using this pattern we obtain a coherent frequency shift as a function of the voltage ratio of

$$
\frac{S}{f_{\mathrm{s}}} \approx 0.303 V_{\mathrm{r}}
$$

According to [5], Landau damping will suppress longitudinal instabilities in the LHC for frequency shifts smaller than

$$
\Delta f_{\text {threshold, Landau }}=0.025 \cdot 23 \mathrm{~Hz}=0.58 \mathrm{~Hz} .
$$

This threshold value gives an intensity safety margin of $41 \%$ for ultimate intensity $\left(N_{\mathrm{b}}=1.7 \times\right.$ $10^{11}$ particles/bunch) and for an impedance of $Z_{L} / n=$ $0.28 \Omega$ at $7 \mathrm{TeV}$.

Operating the LHC with an rf AM, we gain additional longitudinal stability. Keeping a technical margin of 50\%

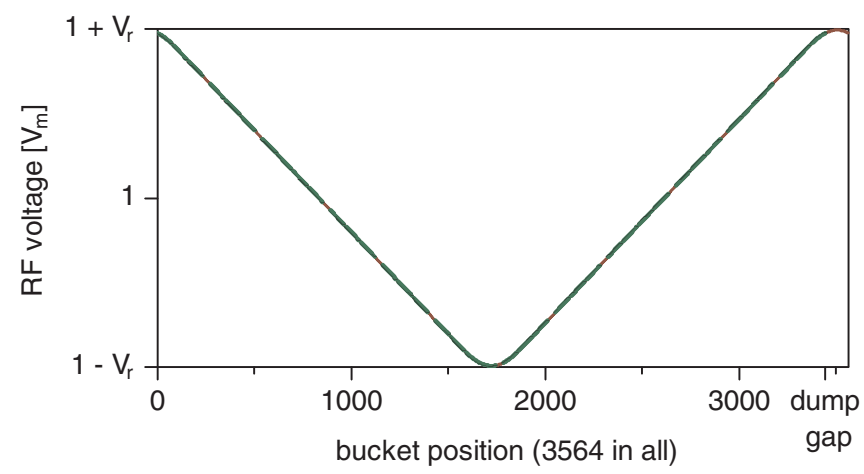

FIG. 14. (Color) Possible rf AM shape for LHC, considering the filling scheme. for transient beam loading compensation, a voltage ratio of $V_{\mathrm{r}}=0.072$ leads to an instability threshold of

$$
\Delta f_{\text {threshold, }} 7.2 \% \mathrm{AM} \approx 0.022 \cdot 23 \mathrm{~Hz}=0.51 \mathrm{~Hz} \text {. }
$$

This increases the total instability threshold to

$\Delta f_{\text {threshold }}=\Delta f_{\text {threshold, Landau }}+\Delta f_{\text {threshold, } \mathrm{AM}} \approx 1.09 \mathrm{~Hz}$.

Accordingly the threshold bunch intensity at which damping is lost is increased from $2.4 \times 10^{11}$ protons per bunch to $4.5 \times 10^{11}$ protons per bunch.

Lowering the nominal voltage from 16 to $14 \mathrm{MV}$ and keeping the technical $50 \%$ safety margin, we increase the AM threshold value to about $0.63 \mathrm{~Hz}$ resulting in a total threshold of $1.14 \mathrm{~Hz}$ and $4.7 \times 10^{11}$ protons per bunch, respectively.

In summary, by operating the LHC with rf AM we double the longitudinal stability.

The rf AM method may also become interesting in case of a demand for shorter bunches for an LHC upgrade. Shorter bunches have inherently less Landau damping and additional stabilization methods would be necessary.

\section{A. Impact of rf AM on luminosity}

At LHC top energy the stable phase $\phi_{\mathrm{s}}$ is $U_{0} /\left(e V_{0}\right)$ [20], with $U_{0}$ the synchrotron radiation loss and $V_{0}$ the peak accelerating voltage, rf AM with $V_{\mathrm{r}}=0.14$ will lead to a modulation of $\phi_{\mathrm{s}}$ by about $14 \%$ or $6.3 \times 10^{-5}$. This means that the bunch position will be modulated by $0.2 \mathrm{ps}$.

Beam loading is another effect to be considered. The LHC rf feedback will not completely cancel beam loading due to limited available rf power. This results in a slight modulation of the bunch positions with respect to the nominal values. This modulation is of the order of $30 \mathrm{ps}$ peak to peak [21]. With the proposed rf AM this modulation will be increased by about $14 \%$ or 4 ps.

Finally, rf AM will modulate the rms bunch length at constant emittance by about $3 \%$ or 8 ps. The rms bunch length of $7.6 \mathrm{~cm}$ is much smaller than the minimum of the beta function of $50 \mathrm{~cm}$. Because of the finite crossing angle small bunch length variations can change the luminosity mainly by virtue of the geometric luminosity reduction factor [22]. A bunch length modulation of 3\% will lead to a luminosity modulation along the bunch train by about $1 \%$. The average change will be zero.

Considering that in physics the expected rms bunch length equals $250 \mathrm{ps}$, rf AM has hardly any influence on the bunch collision points and does not degrade luminosity.

\section{CONCLUSION}

Longitudinal coupled bunch instabilities of LHC type beams in the SPS can be controlled by increasing the bunch to bunch synchrotron frequency spread, in these experiments by modulating the rf amplitude. The achieved stability was comparable to the stability obtained by the $800 \mathrm{MHz}$ Landau damping rf system in normal operation. 
By using the values of the Landau damping system parameters in normal operation we estimated that a voltage ratio of $V_{\mathrm{r}}=0.22$ was needed to obtain the same stability. In our experiments we found that a voltage ratio of $V_{\mathrm{r}}=$ 0.16 was already sufficient to suppress instabilities up to $1.01 \times 10^{11}$ protons per bunch. The difference between these values results certainly from the fact that the $800 \mathrm{MHz}$ values are optimized for accelerating LHC type beams consisting of four batches, whereas we accelerated only one batch. For accelerating four batches, the necessary voltage ratio for the rf AM will be higher.

The results show that a similar method should also work at the LHC if necessary. Using an rf AM at the LHC, we expect to increase the threshold for longitudinal instabilities by a factor of about two, without modification of the rf power amplifiers.

\section{ACKNOWLEDGMENTS}

We would like to thank G. Arduini for measurement time at the SPS. With sincerest appreciation we would also like to thank E. Shaposhnikova, T. Linnecar, and F. Ruggiero for their advice. We want to express our gratitude to J. Tückmantel for examining the technical feasibility of an rf AM at the LHC. Furthermore we are grateful to the shift crews from the SPS and the preaccelerators for supplying good beam conditions.

[1] P. Collier, M. Ainoux, R. Guinand, J-M Jimenez, A. Rizzo, A. Spinks, and K. Weiss, in Proceedings of the 8th European Particle Accelerator Conference, Paris, 2002 (CERN Report No. CERN SL-2002-026 MR, 2002).

[2] T. Bohl, T. Linnecar, and E. Shaposhnikova, in Proceedings of the 8th European Particle Accelerator Conference, Paris, 2002 (EPS-IGA and CERN, Geneva, 2002).

[3] G. Arduini, P. Baudrenghien, T. Bohl, P. Collier, K. Cornelis, W. Höfle, T. Linnecar, E. Shaposhnikova, J. Tuckmantel, and J. Wenninger, in Proceedings of the 8th European Particle Accelerator Conference, Paris, 2002 (Ref. [2]).
[4] T. Bohl, T. Linnecar, E. Shaposhnikova, and J. Tuckmantel, in Proceedings of the European Particle Accelerator Conference, Stockholm, Sweden, 1998 (CERN Report No. SL-98-026 RF, 1998).

[5] D. Boussard, D. Brandt, and L. Vos, CERN, LHC Project Note 205, 1999.

[6] F. J. Sacherer, in Proceedings of the 1973 Particle Accelerator Conference San Francisco, 1973 (CERN Report No. CERN-MPS-Int. BR-73-3, 1973).

[7] S. A. Bogacz and S. Stahl, Fermi National Accelerator Laboratory Report No. FERMILAB-Conf-88/65, 1988; http://fnalpubs.fnal.gov/archive/1988/conf/Conf-88065.pdf

[8] O. Naumann and J. Jacob, in Proceedings of the European Particle Accelerator Conference, Stockholm, Sweden, 1998 (IOP, London, 1998).

[9] E. Vogel, DESY Report No. DESY-HERA-03-03, 2003; http://www.desy.de/ ahluwali/2003h.html

[10] D. Boussard, T. Linnecar, and A. Millich, SPS Commissioning Report No. 20, 1973; CERN Report No. CERN-SPS-Com-Rep-76-020, 1976.

[11] G. Dôme, CERN Report No. CERN-SPS-ARF-77-11, 1977.

[12] CERN Photo No. CERN-AC-7802669, 1978.

[13] CERN Photo No. CERN-AC-7902734, 1979.

[14] E. Shaposhnikova (private communication); she provided us with these values, CERN, 2003.

[15] P. Baudrenghien and G. Lambert, in Proceedings of Chamonix X, 2000 (CERN Report No. CERN-SL-2000007, 2000).

[16] P. Baudrenghien, in Proceedings of Chamonix IX, 1999 (CERN Report No. CERN-SL-99-007 DI, 1999).

[17] E. Vogel, personal observations at the HERA proton ring, 2003.

[18] J. Tückmantel (private communication).

[19] J. Tückmantel, CERN, AB Note 2004-045 RF, 2004.

[20] E. Shaposhnikova, S. Fartoukh, and B. Jeanneret, in Proceedings of the 9th European Particle Accelerator Conference, Lucerne, Switzerland (CERN Report No. LHC-Project-Report-763, 2004).

[21] J. Tückmantel, CERN, AB Note 2004-022 RF, 2004.

[22] LHC Design Report, edited by O. Brüning, P. Collier, P. Lebrun, S. Myers, R. Ostojic , J. Poole, P. Proudlock, Vol. 1: CERN Report No. CERN-2004-003, 2004. 